\title{
Single-nucleotide polymorphisms in PSCA and the risk of breast cancer in a Chinese population
}

\author{
Meng Wang ${ }^{1}$, Xijing Wang ${ }^{1}$, Sidney W. Fư ${ }^{2}$, Xinghan Liu ${ }^{1}$, Tianbo Jin ${ }^{3}$, Huafeng \\ Kang $^{1}$, Xiaobin Ma ${ }^{1}$, Shuai Lin ${ }^{1}$, Haitao Guan ${ }^{1}$, Shuqun Zhang ${ }^{1}$, Kang Liu ${ }^{1}$, Cong \\ Dai ${ }^{1}$, Yuyao Zhu ${ }^{1}$, Zhijun Dai' ${ }^{1,2}$ \\ ${ }^{1}$ Department of Oncology, Second Affiliated Hospital of Xi'an Jiaotong University, Xi'an 710004, China \\ ${ }^{2}$ Division of Genomic Medicine/Department of Medicine, The George Washington University School of Medicine and Health \\ Sciences, Washington, DC 20037, USA \\ ${ }^{3}$ National Engineering Research Center for Miniaturized Detection Systems, School of Life Sciences, Northwest University, \\ Xi'an 710069, China
}

Correspondence to: ZhiJun Dai, e-mail: dzj0911@mail.xjtu.edu.cn; dzj0911@126.com

Keywords: PSCA, single-nucleotide polymorphisms, breast cancer, susceptibility

Received: December 07, $2015 \quad$ Accepted: March 18, $2016 \quad$ Published: March 30, 2016

\section{ABSTRACT}

This study explored the associations between common PSCA single-nucleotide polymorphisms (rs2294008, rs2978974, and rs2976392) and breast cancer among 560 breast cancer cases and 583 controls (Chinese Han women). We found rs2294008 was significantly associated with a high risk of breast cancer (homozygote model, odds ratio [OR]: 1.67, 95\% confidence interval [CI]: 1.06-2.59; recessive, OR: 1.64, 95\% CI: 1.06-2.53). And stratification by menopausal status revealed an association of the minor allele of rs2294008 with breast cancer risk among premenopausal (homozygote model, OR: 2.41, 95\% CI: 1.03-5.66; recessive, OR: 2.80, $95 \%$ CI: 1.21-6.47) and postmenopausal women (allele model, OR: 1.29, 95\% CI: 1.01-1.65). Rs2978974 influenced the breast cancer risk among postmenopausal women in heterozygote model (OR: 1.47, 95\% CI: 1.05-2.07). When stratified by clinicopathologic features, the T allele of rs2294008 was associated with progesterone receptor status (homozygote model, OR: 1.98, 95\% CI: 1.08-3.63; recessive, OR: 1.87, 95\% CI: 1.04-3.37), and the rs2976392 polymorphism was associated with high lymph node metastasis risk in homozygote model (OR: 2.09, 95\%CI: 1.014.31). Further haplotype analysis suggested that $T_{r s 2294008} A_{r s 2976392} G_{r s 2978974}$ haplotype enhances breast cancer risk (OR:1.52, 95\%CI:1.23-1.89, $P<0.001$ ). Therefore, among Chinese Han women, the PSCA rs2294008, rs2978974, and rs2976392 minor alleles are associated with increased breast cancer risk especially in progesterone receptor positive breast cancer patients, with breast cancer risk in postmenopausal women, and with high lymph node metastasis risk, respectively. Moreover, $T_{\text {rs2294008 }} A_{r s 2976392}$ $\mathbf{G}_{\text {rs2978974 }}$ haplotype was associated with significantly increased risk of breast cancer.

\section{INTRODUCTION}

Breast cancer is the most common cancer and the principal cause of cancer-related deaths among Chinese women [1], accounted for 248,620 new cases and 60,473 cancer-related deaths during 2011 [2]. It is a multifactorial disease influenced by complex interactions between genetic, environmental, and lifestyle factors [3]. Genetic research provides insight into carcinogenesis, including the development and treatment of breast cancer.
Single-nucleotide polymorphisms (SNPs) are variations in a single base pair in the DNA sequence and have been widely studied in cancer research in recent years. Several genes that affect breast cancer risk, including $B R C A 1$ (breast cancer 1), BRCA2 (breast cancer 2), PTEN (phosphatase and tensin homolog deleted on chromosome ten), and TP53 (tumor protein p53) have been identified [4-8].

PSCA encodes a 123-amino acid immature lymphocyte cell surface maker with $30 \%$ homology to stem cell antigen 
type 2, a member of the Thy-1/Ly-6 family and is located on chromosome 8q24.2 [10]. PSCA was initially identified as a prostate-specific antigen over-expressed in $>80 \%$ of prostate cancers, including metastatic and hormone-related cancers $[10,11]$. Recent studies have shown that PSCA is also abnormally expressed in bladder cancer [12-14], gastric cancer [15-17], renal cell carcinoma [18], oesophageal cancer [19], gallbladder cancer [20-22], and pancreatic cancer [23]. Studies in vitro indicated that $P S C A$ being transfected into PSCA-negative cells caused down-regulated cell proliferation, thus affecting survival of gastric cancer cells [24]. And, down-regulation of PSCA in a human bladder cancer cell line led to inhibition of cell growth via activation of several immune signaling pathways [25]. Genome-wide association studies have revealed many PSCA polymorphisms, among which rs2294008 C > T, rs2978974 $\mathrm{G}>\mathrm{A}$, and rs2976392 $\mathrm{G}>\mathrm{A}$ are the most widely studied ones and may influence susceptibility to different types of cancer $[22,26,27]$. However, few studies have been performed to investigate the associations of these three PSCA SNPs with breast cancer. A single study with small sample sizes (456 patients and 461controls) revealed that the PSCA SNPs were associated with breast cancer susceptibility among Korean women [9]. Therefore, the present study aimed to comprehensively examine the potential association of three SNPs (rs2294008 C>T, rs2978974 G>A, and rs2976392 $\mathrm{G}>\mathrm{A}$ ) in $P S C A$ with the risk of breast cancer among a population of Chinese women.

\section{RESULTS}

\section{Associations between PSCA SNPs and the risk of breast cancer}

Detailed allele frequencies and genotype distributions of the three polymorphisms are shown in Table 1. The distributions of rs2294008, rs2978974, and rs2976392 in the control group were in accordance with Hardy-Weinberg equilibrium $(P=0.195, P=0.164$, and $P$ $=0.179$, respectively). Both the homozygote and recessive models of rs2294008 revealed an associated with a high risk of breast cancer (TT vs. CC, odds ratio [OR]: 1.67, 95\% confidence interval $[\mathrm{CI}]: 1.06-2.59, P=0.03$; TT vs. CC+TC, OR: $1.64,95 \% \mathrm{CI}: 1.06-2.53, P=0.02)$. We further calculated the power of the rs2294008 SNP homozygote and recessive model analyses, and we were able to reject the null hypothesis that the TT frequency for case and controls is equal with probability (power) $=0.896$. No significant associations with rs2976392 and rs2978974 were found in any of the models.

\section{Subgroup analyses according to age and menopausal status}

Stratification analyses according to age revealed no significant associations between the three PSCA SNPs and the risk of breast cancer (all, $P>0.05$ ) (Table 2). While, stratification analyses according to menopausal status (Table 3) found that the minor allele of rs2294008 was a risk factor among both premenopausal women (homozygote model, OR: 2.41, 95\% CI: 1.03-5.66, $P=0.04$; recessive model, OR: $2.80,95 \% \mathrm{CI}: 1.21-6.47$, $P=0.01$ ) and postmenopausal women (allele model: OR: 1.29, 95\% CI: $1.01-1.65, P=0.04)$. For rs2978974, a significant association with high breast cancer risk was found among postmenopausal women in the heterozygote model (OR: 1.47, 95\% CI: 1.05-2.07, $P=0.03$ ). There were no significant associations with rs2976392 in any of the subgroups.

\section{Associations between PSCA SNPs and the clinicopathological features of breast cancer}

We evaluated the associations of PSCA SNPs with various clinicopathological features including: tumor size, lymph node metastasis, and the expressions of estrogen receptor (ER), progesterone receptor (PR), and human epidermal growth factor receptor 2 (HER-2). The T allele of rs2294008 was associated with positive PR status (homozygote model, OR: 1.98, 95\% CI: 1.08-3.63, $P=0.03$; recessive model, OR: $1.87,95 \% \mathrm{CI}: 1.04-3.37$, $P=0.03$ ) (Table 4). The minor allele of rs2976392 was associated with a high risk of lymph node metastasis in the homozygote model (OR: 2.09, 95\% CI: 1.01-4.31, $P=0.04)$. However, rs 2978974 was not significantly associated with any of the clinicopathological features.

\section{Association between $P S C A$ haplotypes and breast cancer risk}

We analyzed the association between PSCA haplotypes and the risk of breast cancer. Table 5 shows that $\mathrm{T}_{\mathrm{rs} 2294008} \mathrm{~A}_{\mathrm{rs} 2976392} \mathrm{G}_{\mathrm{rs} 2978974}$ haplotype was associated with a significantly increased risk of breast cancer (OR: 1.52, 95\%CI: 1.23-1.89, $P<0.001$ ). The "others" (haplotypes with frequency $<1 \%$ were merged) were broadly distributed in cases at a low level (OR: 0.46, 95\%CI: 0.29$0.71, P<0.001)$. The significance of this result is limited given the naturally low frequencies of these haplotypes. We did not discover any associations with $\mathrm{C}_{\mathrm{rs} 2294008} \mathrm{G}_{\mathrm{rs} 2976392}$ $\mathrm{A}_{\mathrm{rs} 2978974}$ and $\mathrm{T}_{\mathrm{rs} 2294008} \mathrm{~A}_{\mathrm{rs} 2976392} \mathrm{~A}_{\mathrm{rs} 2978974}$ in breast cancer.

\section{DISCUSSION}

Genetic studies have provided insight into various diseases, including cancers. Understanding the associations between different genes and cancers can improve prevention, treatment, and prognosis estimation. Genome-wide association studies have revealed many genetic markers of different cancers. Recently numerous studies have indicated that PSCA may influence a diverse group of cancers, including gastric, bladder, renal, and 
Table 1: Genotype frequencies of $P S C A$ polymorphisms in cases and controls

\begin{tabular}{|c|c|c|c|c|c|}
\hline Model & Genotype & Cases $(n, \%)$ & Control (n,\%) & $\boldsymbol{P}^{\dagger}$ & OR $(95 \% \mathrm{CI})$ \\
\hline rs2294008 & \multicolumn{5}{|c|}{ HWE: $P=0.195$} \\
\hline Co-dominant & $\mathrm{CC}$ & $273(48.8 \%)$ & $299(51.3 \%)$ & & \\
\hline Heterozygote & $\mathrm{TC}$ & $231(41.3 \%)$ & $247(42.4 \%)$ & 0.85 & $1.02(0.80-1.31)$ \\
\hline Homozygote & $\mathrm{TT}$ & $56(10.0 \%)$ & $37(6.3 \%)$ & 0.03 & $1.67(1.06-2.59)$ \\
\hline \multirow[t]{2}{*}{ Dominant } & $\mathrm{CC}$ & $273(48.8 \%)$ & $299(51.3 \%)$ & & \\
\hline & $\mathrm{TC}+\mathrm{TT}$ & $287(51.3 \%)$ & $284(48.7 \%)$ & 0.39 & $1.11(0.88-1.40)$ \\
\hline \multirow[t]{2}{*}{ Recessive } & $\mathrm{CC}+\mathrm{TC}$ & $504(90.0 \%)$ & $546(93.7 \%)$ & & \\
\hline & TT & $56(10.0 \%)$ & $37(6.3 \%)$ & 0.02 & $1.64(1.06-2.53)$ \\
\hline \multirow[t]{2}{*}{ Overdominant } & $\mathrm{CC}+\mathrm{TT}$ & $329(\%)$ & $336(57.6 \%)$ & & \\
\hline & $\mathrm{TC}$ & $231(\%)$ & $247(42.4 \%)$ & 0.70 & $0.96(0.76-1.21)$ \\
\hline \multirow[t]{2}{*}{ Allele } & $\mathrm{C}$ & $777(69.4 \%)$ & $845(72.5 \%)$ & & \\
\hline & $\mathrm{T}$ & $343(30.6 \%)$ & $321(27.5 \%)$ & 0.10 & $1.16(0.97-1.39)$ \\
\hline rs2976392 & \multicolumn{5}{|c|}{ HWE: $P=0.164$} \\
\hline Co-dominant & GG & $287(51.3 \%)$ & $298(51.1 \%)$ & & \\
\hline Heterozygote & GA & $230(41.1 \%)$ & $247(42.4 \%)$ & 0.79 & $0.97(0.76-1.23)$ \\
\hline Homozygote & $\mathrm{AA}$ & $43(7.7 \%)$ & $38(6.5 \%)$ & 0.50 & $1.18(0.74-1.87)$ \\
\hline \multirow[t]{2}{*}{ Dominant } & GG & $287(51.3 \%)$ & $298(51.1 \%)$ & & \\
\hline & $\mathrm{GA}+\mathrm{AA}$ & $273(48.8 \%)$ & $285(48.9 \%)$ & 0.96 & $1.00(0.79-1.25)$ \\
\hline \multirow[t]{2}{*}{ Recessive } & $\mathrm{GG}+\mathrm{GA}$ & $517(92.3 \%)$ & $545(93.5 \%)$ & & \\
\hline & $\mathrm{AA}$ & $43(7.7 \%)$ & $38(6.5 \%)$ & 0.45 & $1.19(0.76-1.88)$ \\
\hline \multirow[t]{2}{*}{ Overdominant } & $\mathrm{GG}+\mathrm{AA}$ & $330(58.9 \%)$ & $336(57.6 \%)$ & & \\
\hline & GA & $230(41.1 \%)$ & $247(42.4 \%)$ & 0.66 & $0.95(0.75-1.20)$ \\
\hline \multirow[t]{2}{*}{ Allele } & $\mathrm{G}$ & $804(71.8 \%)$ & $843(72.3 \%)$ & & \\
\hline & A & $316(28.2 \%)$ & $323(27.7 \%)$ & 0.79 & $1.03(0.85-1.23)$ \\
\hline rs2978974* & \multicolumn{5}{|c|}{ HWE: $P=0.179$} \\
\hline Co-dominant & GG & $254(45.4 \%)$ & $283(48.5 \%)$ & & \\
\hline Heterozygote & GA & $259(46.3 \%)$ & $256(43.9 \%)$ & 0.33 & $1.13(0.89-1.44)$ \\
\hline Homozygote & $\mathrm{AA}$ & $46(8.2 \%)$ & $44(7.5 \%)$ & 0.50 & $1.17(0.75-1.82)$ \\
\hline \multirow[t]{2}{*}{ Dominant } & GG & $254(45.4 \%)$ & $283(48.5 \%)$ & & \\
\hline & $\mathrm{GA}+\mathrm{AA}$ & $305(54.6 \%)$ & $300(51.5 \%)$ & 0.29 & $1.13(0.90-1.43)$ \\
\hline \multirow[t]{2}{*}{ Recessive } & $\mathrm{GG}+\mathrm{GA}$ & $513(91.8 \%)$ & $539(92.5 \%)$ & & \\
\hline & AA & $46(8.2 \%)$ & $44(7.5 \%)$ & 0.67 & $1.10(0.71-1.69)$ \\
\hline \multirow[t]{2}{*}{ Overdominant } & $\mathrm{GG}+\mathrm{AA}$ & $300(53.7 \%)$ & $327(56.1 \%)$ & & \\
\hline & GA & $259(46.3 \%)$ & $256(43.9 \%)$ & 0.41 & $1.10(0.87-1.39)$ \\
\hline \multirow[t]{2}{*}{ Allele } & G & $767(68.6 \%)$ & $822(70.5 \%)$ & & \\
\hline & A & $351(31.4 \%)$ & $344(29.0 \%)$ & 0.33 & $1.09(0.92-1.31)$ \\
\hline
\end{tabular}

OR: odds ratio; 95\%CI: confidence interval.

* Cases of rs2978974 polymorphism missing $n=1$

${ }^{\dagger}$ Adjusted for age and body mass index. 
Table 2: Association between PSCA SNPs and age of breast cancer patients

\begin{tabular}{|c|c|c|c|c|c|c|c|c|c|c|c|}
\hline \multirow[t]{2}{*}{ Age(years) } & \multicolumn{3}{|c|}{$\begin{array}{c}\text { genotype } \\
\text { distributions(case/control) }\end{array}$} & \multicolumn{2}{|r|}{ Co-dominant } & \multicolumn{2}{|c|}{ Dominant } & \multicolumn{2}{|c|}{ Recessive } & \multicolumn{2}{|r|}{ Allele } \\
\hline & $\mathbf{A A}$ & Aa & aa & $P$ & OR $(95 \% C I)$ & $P$ & $\begin{array}{c}\text { OR } \\
(95 \% \mathrm{CI})\end{array}$ & $P$ & $\begin{array}{c}\text { OR } \\
(95 \% \mathrm{CI})\end{array}$ & $P$ & $\begin{array}{c}\text { OR } \\
(95 \% \mathrm{CI})\end{array}$ \\
\hline & \multicolumn{11}{|c|}{ rs2294008 } \\
\hline$<49$ & $135 / 157$ & $128 / 131$ & $31 / 23$ & $0.46^{\mathrm{m}}$ & $\begin{array}{l}1.14(0.81-1.59)^{\mathrm{m}} \\
1.57(0.87-2.82)^{\mathrm{n}}\end{array}$ & 0.26 & $\begin{array}{c}1.20 \\
(0.87-1.65)\end{array}$ & 0.18 & $\begin{array}{c}1.48 \\
(0.84-2.60)\end{array}$ & 0.15 & $\begin{array}{c}1.20 \\
(0.94-1.53)\end{array}$ \\
\hline \multirow[t]{2}{*}{$\geq 49$} & $138 / 142$ & $103 / 116$ & $25 / 14$ & $\begin{array}{l}0.62^{\mathrm{m}} \\
0.08^{\mathrm{n}}\end{array}$ & $\begin{array}{l}0.91(0.64-1.30)^{\mathrm{m}} \\
1.84(0.92-3.68)^{\mathrm{n}}\end{array}$ & 0.94 & $\begin{array}{c}1.01 \\
(0.72-1.42)\end{array}$ & 0.06 & $\begin{array}{c}1.91 \\
(0.97-3.76)\end{array}$ & 0.40 & $\begin{array}{c}1.12 \\
(0.86-1.47)\end{array}$ \\
\hline & \multicolumn{11}{|c|}{ rs2976392 } \\
\hline$<49$ & $156 / 163$ & $117 / 130$ & $21 / 18$ & $\begin{array}{l}0.72^{\mathrm{m}} \\
0.56^{\mathrm{n}}\end{array}$ & $\begin{array}{l}0.94(0.67-1.31)^{\mathrm{m}} \\
1.22(0.63-2.37)^{\mathrm{n}}\end{array}$ & 0.87 & $\begin{array}{c}0.97 \\
(0.71-1.34)\end{array}$ & 0.50 & $\begin{array}{c}1.25 \\
(0.65-2.40)\end{array}$ & 0.89 & $\begin{array}{c}1.02 \\
(0.79-1.31)\end{array}$ \\
\hline \multirow[t]{2}{*}{$\geq 49$} & $131 / 135$ & $113 / 117$ & $22 / 20$ & $0.98^{\mathrm{m}}$ & $1.00(0.70-1.42)^{\mathrm{m}}$ & 0.93 & $\begin{array}{c}1.02 \\
(0.72-1.42)\end{array}$ & 0.90 & $\begin{array}{c}0.98 \\
(0.70-1.38)\end{array}$ & 0.81 & $\begin{array}{c}1.03 \\
(0.79-1.34)\end{array}$ \\
\hline & \multicolumn{11}{|c|}{ rs2978974 } \\
\hline$<49$ & $135 / 159$ & $142 / 138$ & $17 / 14$ & $\begin{array}{l}0.25^{\mathrm{m}} \\
0.34^{\mathrm{n}}\end{array}$ & $\begin{array}{l}1.21(0.87-1.68)^{\mathrm{m}} \\
1.43(0.68-3.01)^{\mathrm{n}}\end{array}$ & 0.20 & $\begin{array}{c}1.23 \\
(0.90-1.70)\end{array}$ & 0.48 & $\begin{array}{c}1.30 \\
(0.63-2.69)\end{array}$ & 0.21 & $\begin{array}{c}1.17 \\
(0.91-1.51)\end{array}$ \\
\hline$\geq 49$ & $119 / 124$ & $117 / 118$ & $29 / 30$ & $0.86^{\mathrm{m}}$ & $\begin{array}{l}1.03(0.72-1.48)^{\mathrm{m}} \\
1.01(0.57-1.78)^{\mathrm{n}}\end{array}$ & 0.87 & $\begin{array}{c}1.03 \\
(0.73-1.44)\end{array}$ & 0.98 & $\begin{array}{c}1.00 \\
(0.58-1.70)\end{array}$ & 0.92 & $\begin{array}{c}1.01 \\
(0.79-1.31)\end{array}$ \\
\hline
\end{tabular}

A: Major allele; a: Minor allele; $m=$ Heterozygote model; $n=$ Homozygote model; OR: odds ratio; $95 \%$ CI: confidence interval.

pancreatic cancers $[9,12-23,26]$. However, there is little insight into the relationship between PSCA and breast cancer.

Rs2294008 is located in exon 1 of PSCA and its C to $\mathrm{T}$ transition has been shown to reduce transcriptional activity of an upstream fragment of PSCA [28, 29]. Precious meta-analyses discovered that $\mathrm{T}$ allele of rs2294008 was a risk factor for cancer, particularly for gastric and bladder cancers $[26,27]$. The $\mathrm{T}$ allele of rs2294008 increased risk for gastric cancer in Asian populations $[30,31]$ and the genetic variant rs 2294008 was identified to confer genetic susceptibility for bladder cancer risk in both Caucasian [12] and Asian [14, 20] populations. In this study, we found that the minor allele of rs2294008 was associated with a high risk of breast cancer among both premenopausal and postmenopausal women. There was no association between rs2294008 and ER status, although PR-positive tumors were associated with the $\mathrm{T}$ allele. In contrast, a study based on Korean women reported that the minor allele of rs2294008 was associated with reduced breast cancer risk among premenopausal women, increased breast cancer risk among postmenopausal women, and that the $\mathrm{T}$ allele increased the ER-negative breast cancer risk [9]. Whist similar, our study provides a more robust analysis as it includes more patients as well as more detailed stratified analyses. Given the heterogeneous nature of breast cancer, the discrepancies between our findings and those of Kim et al. [9] may be explained by various factors, including region, lifestyle, genetic testing methods, and study design.

Rs2976392 is located in the intron 2 of PSCA and has a strong linkage disequilibrium with rs2294008 $\mathrm{C}>\mathrm{T}[24,32]$. The association of this SNP and cancer susceptibility has been widely investigated. Recent metaanalysis has revealed the PSCA rs2976392 polymorphism was significantly associated with increased overall cancer risk [27]. Rs 2978974 in the promoter region of PSCA showed low linkage disequilibrium with rs2294008 and the $\mathrm{A}_{\mathrm{r} 22978974}$ allele was shown to contribute to bladder cancer susceptibility, presumably due to the loss of binding of ELK1 or other ETS proteins to the PSCA promoter [12]. A study based on 405 gallbladder cancer patients and 247 healthy controls showed that the PSCA haplotype $\mathrm{T}_{\mathrm{r} 2224008}$ $\mathrm{A}_{\mathrm{r} 22978974}$ conferred low risk of gallbladder cancer in males, while in females, the $T_{\mathrm{rs} 2294008} G_{\mathrm{r} 22978974}$ haplotype was related to increased gallbladder cancer risk [22]. Kim et al. found that there was no statistically significant relationship between rs2976392 and breast cancer risk, which is concordant with our study. However, we found the rs2976392 SNP was 
Table 3: Association between PSCA SNPs and menopausal status of breast cancer patients

\begin{tabular}{|c|c|c|c|c|c|c|c|c|c|c|c|}
\hline \multirow[t]{2}{*}{$\begin{array}{l}\text { menopausal } \\
\text { status }\end{array}$} & \multicolumn{3}{|c|}{$\begin{array}{c}\text { genotype distributions } \\
\text { (case/control) }\end{array}$} & \multicolumn{2}{|c|}{ Co-dominant } & \multicolumn{2}{|c|}{ Dominant } & \multicolumn{2}{|c|}{ Recessive } & \multicolumn{2}{|r|}{ Allele } \\
\hline & $\mathbf{A A}$ & Aa & aa & $\boldsymbol{P}$ & OR (95\%CI) & $P$ & $\begin{array}{c}\text { OR } \\
(95 \% \mathrm{CI})\end{array}$ & $P$ & $\begin{array}{c}\text { OR } \\
(95 \% \mathrm{CI})\end{array}$ & $P$ & $\begin{array}{c}\text { OR } \\
(95 \% \mathrm{CI})\end{array}$ \\
\hline & \multicolumn{11}{|c|}{ rs2294008 } \\
\hline Premenopausal & $143 / 138$ & $101 / 135$ & $20 / 8$ & $\begin{array}{l}0.07^{\mathrm{m}} \\
\mathbf{0 . 0 4 ^ { \mathrm { n } }}\end{array}$ & $0.72(0.51-1.02)^{\mathrm{m}}$ & 0.24 & $\begin{array}{c}0.82 \\
(0.58-1.13)\end{array}$ & 0.01 & $\begin{array}{c}2.80 \\
(1.21-6.47)\end{array}$ & 0.95 & $\begin{array}{c}0.99 \\
(0.76-1.30)\end{array}$ \\
\hline \multirow[t]{2}{*}{ Postmenopausal } & $130 / 161$ & $120 / 112$ & $36 / 29$ & $0.11^{\mathrm{m}}$ & $1.33(0.94-1.88)^{\mathrm{m}}$ & 0.06 & $\begin{array}{c}1.37 \\
(0.99-1.90)\end{array}$ & 0.25 & $\begin{array}{c}1.36 \\
(0.81-2.28)\end{array}$ & 0.04 & $\begin{array}{c}1.29 \\
(1.01-1.65)\end{array}$ \\
\hline & \multicolumn{11}{|l|}{ rs2976392 } \\
\hline Premenopausal & $131 / 140$ & $118 / 129$ & $15 / 12$ & $0.47^{\mathrm{n}}$ & $\begin{array}{l}0.98(0.69-1.38)^{\mathrm{m}} \\
1.34(0.60-2.96)^{\mathrm{n}}\end{array}$ & 0.96 & $\begin{array}{c}1.01 \\
(0.72-1.41)\end{array}$ & 0.45 & $\begin{array}{c}1.35 \\
(0.62-2.94)\end{array}$ & 0.77 & $\begin{array}{c}1.04 \\
(0.80-1.36)\end{array}$ \\
\hline \multirow[t]{2}{*}{ Postmenopausal } & $156 / 158$ & $112 / 118$ & $28 / 26$ & $0.82^{\mathrm{m}}$ & $0.96(0.68-1.35)^{\mathrm{m}}$ & 0.93 & $\begin{array}{c}0.99 \\
(0.71-1.36)\end{array}$ & 0.72 & $\begin{array}{c}1.11 \\
(0.63-1.94)\end{array}$ & 0.93 & $\begin{array}{c}1.01 \\
(0.79-1.30)\end{array}$ \\
\hline & \multicolumn{11}{|c|}{ rs2978974 } \\
\hline Premenopausal & $129 / 131$ & $115 / 137$ & $20 / 13$ & $0.37^{\mathrm{m}}$ & $0.85(0.60-1.21)^{\mathrm{m}}$ & 0.60 & $\begin{array}{c}0.91 \\
(0.65-1.28)\end{array}$ & 0.15 & $\begin{array}{c}1.69 \\
(0.82-3.47)\end{array}$ & 0.90 & $\begin{array}{c}1.02 \\
(0.78-1.32)\end{array}$ \\
\hline & & & & $\mathbf{0 . 0 3}^{\mathrm{m}}$ & $1.47(1.05-2.07)^{\mathrm{m}}$ & & 1.38 & & 0.85 & & 1.16 \\
\hline Postmenopausal & $125 / 152$ & $144 / 119$ & $26 / 31$ & $0.95^{\mathrm{n}}$ & $1.02(0.58-1.81)^{\mathrm{n}}$ & 0.05 & $(1.00-1.90)$ & 0.55 & $(0.49-1.46)$ & 0.23 & $(0.91-1.48)$ \\
\hline
\end{tabular}

A: Major allele; a: Minor allele; m= Heterozygote model; $\mathrm{n}=$ Homozygote model; OR: odds ratio; 95\%CI: confidence interval.

Table 4: The associations between the PSCA polymorphisms and clinical characteristics of breast cancer patients

\begin{tabular}{|c|c|c|c|c|c|c|c|c|c|c|c|}
\hline \multirow[t]{2}{*}{ Variables } & \multirow[t]{2}{*}{$\mathbf{A A}$} & \multirow[t]{2}{*}{ Aa } & \multirow[t]{2}{*}{ aa } & \multicolumn{2}{|c|}{ Co-dominant } & \multicolumn{2}{|c|}{ Dominant } & \multicolumn{2}{|c|}{ Recessive } & \multicolumn{2}{|r|}{ Allele } \\
\hline & & & & $P$ & OR $(95 \% C I)$ & $P$ & $\begin{array}{c}\text { OR } \\
(95 \% \mathrm{CI})\end{array}$ & $P$ & $\begin{array}{c}\text { OR } \\
(95 \% \mathrm{CI})\end{array}$ & $P$ & $\begin{array}{c}\text { OR } \\
(95 \% \mathrm{CI})\end{array}$ \\
\hline \multicolumn{12}{|c|}{ rs2294008 } \\
\hline \multicolumn{12}{|c|}{ Tumor size } \\
\hline$<2 \mathrm{~cm}$ & 98 & 75 & 15 & \multicolumn{2}{|c|}{1.00 (reference) } & & & & & & \\
\hline$\geq 2 \mathrm{~cm}$ & 175 & 156 & 41 & $0.42^{\mathrm{m}}$ & $1.17(0.80-1.69)^{\mathrm{m}}$ & 0.26 & $\begin{array}{c}1.23 \\
(0.86-1.74)\end{array}$ & 0.26 & $\begin{array}{c}1.43 \\
(0.77-2.65)\end{array}$ & 0.16 & $\begin{array}{c}1.21 \\
(0.92-1.60)\end{array}$ \\
\hline \multicolumn{12}{|c|}{ LN metastasis } \\
\hline Negative & 109 & 98 & 29 & \multicolumn{2}{|c|}{1.00 (reference) } & & & & & & \\
\hline Positive & 164 & 133 & 27 & $1.10^{\mathrm{n}}$ & $0.62(0.35-1.10)^{\mathrm{n}}$ & 0.30 & $\begin{array}{c}0.84 \\
(0.60-1.17)\end{array}$ & 1.12 & $\begin{array}{c}0.65 \\
(0.37-1.13)\end{array}$ & 1.13 & $\begin{array}{c}0.82 \\
(0.64-1.06)\end{array}$ \\
\hline \multicolumn{12}{|l|}{ ER } \\
\hline Negative & 124 & 110 & 13 & \multicolumn{2}{|c|}{1.00 (reference) } & & & & & & \\
\hline Positive & 143 & 149 & 21 & $0.36^{\mathrm{m}}$ & $\begin{array}{l}1.18(0.83-1.66)^{\mathrm{m}} \\
1.40(0.67-2.91)^{\mathrm{n}}\end{array}$ & 0.29 & $\begin{array}{c}1.20 \\
(0.86-1.67)\end{array}$ & 0.48 & $\begin{array}{c}1.30 \\
(0.64-2.64)\end{array}$ & 0.28 & $\begin{array}{c}1.16 \\
(0.89-1.50)\end{array}$ \\
\hline
\end{tabular}

(Continued) 


\begin{tabular}{|c|c|c|c|c|c|c|c|c|c|c|c|}
\hline \multirow[t]{2}{*}{ Variables } & \multirow[t]{2}{*}{$\mathbf{A A}$} & \multirow[t]{2}{*}{ Aa } & \multirow[t]{2}{*}{ aa } & \multicolumn{2}{|c|}{ Co-dominant } & \multicolumn{2}{|c|}{ Dominant } & \multicolumn{2}{|c|}{ Recessive } & \multicolumn{2}{|r|}{ Allele } \\
\hline & & & & $P$ & OR $(95 \% \mathrm{CI})$ & $P$ & $\begin{array}{c}\text { OR } \\
(95 \% \mathrm{CI})\end{array}$ & $P$ & $\begin{array}{c}\text { OR } \\
(95 \% \mathrm{CI})\end{array}$ & $P$ & $\begin{array}{c}\text { OR } \\
(95 \% \mathrm{CI})\end{array}$ \\
\hline \multicolumn{12}{|l|}{ PR } \\
\hline Negative & 132 & 105 & 18 & \multicolumn{2}{|c|}{1.00 (reference) } & & & & & & \\
\hline Positive & 141 & 126 & 38 & $0.52^{\mathrm{m}}$ & $\begin{array}{l}1.12(0.79-1.60)^{\mathrm{m}} \\
1.98(1.08-3.63)^{\mathrm{n}}\end{array}$ & 0.19 & $\begin{array}{c}1.25 \\
(0.90-1.74)\end{array}$ & 0.03 & $\begin{array}{c}1.87 \\
(1.04-3.37)\end{array}$ & 0.05 & $\begin{array}{c}1.30 \\
(1.00-1.68)\end{array}$ \\
\hline \multicolumn{12}{|l|}{ HER-2 } \\
\hline Negative & 190 & 166 & 33 & \multicolumn{2}{|c|}{1.00 (reference) } & & & & & & \\
\hline Positive & 83 & 65 & 23 & $0.58^{\mathrm{m}}$ & $0.90(0.61-1.32)^{\mathrm{m}}$ & 0.95 & $\begin{array}{c}1.01 \\
(0.71-1.45)\end{array}$ & 0.52 & $\begin{array}{c}1.21 \\
(0.68-2.13)\end{array}$ & 0.38 & $\begin{array}{c}1.13 \\
(0.86-1.49)\end{array}$ \\
\hline \multicolumn{12}{|l|}{ rs2976392 } \\
\hline \multicolumn{12}{|c|}{ Tumor size } \\
\hline$<2 \mathrm{~cm}$ & 97 & 78 & 13 & \multicolumn{2}{|c|}{1.00 (reference) } & & & & & & \\
\hline$\geq 2 \mathrm{~cm}$ & 190 & 152 & 30 & $0.64^{\mathrm{n}}$ & $\begin{array}{l}1.00(0.69-1.44)^{\mathrm{m}} \\
1.18(0.59-2.36)^{\mathrm{n}}\end{array}$ & 0.91 & $\begin{array}{c}1.02 \\
(0.72-1.45)\end{array}$ & 0.63 & $\begin{array}{c}1.18 \\
(0.60-2.32)\end{array}$ & 0.77 & $\begin{array}{c}1.04 \\
(0.79-1.37)\end{array}$ \\
\hline \multicolumn{12}{|c|}{ LN metastasis } \\
\hline Negative & 120 & 105 & 11 & \multicolumn{2}{|c|}{1.00 (reference) } & & & & & & \\
\hline Positive & 167 & 125 & 32 & $0.38^{\mathrm{m}}$ & $0.86(0.60-1.21)^{\mathrm{m}}$ & 0.87 & $\begin{array}{c}0.97 \\
(0.70-1.36)\end{array}$ & 0.15 & $\begin{array}{c}1.67 \\
(0.83-3.38)\end{array}$ & 0.41 & $\begin{array}{c}1.12 \\
(0.86-1.46)\end{array}$ \\
\hline \multicolumn{12}{|l|}{ ER } \\
\hline Negative & 125 & 99 & 23 & \multicolumn{2}{|c|}{1.00 (reference) } & & & & & & \\
\hline Positive & 162 & 131 & 20 & $0.91^{\mathrm{m}}$ & $0.67(0.35-1.28)^{\mathrm{n}}$ & 0.79 & $\begin{array}{c}0.96 \\
(0.68-1.33)\end{array}$ & 0.20 & $\begin{array}{c}0.67 \\
(0.36-1.24)\end{array}$ & 0.45 & $\begin{array}{c}0.91 \\
(0.70-1.18)\end{array}$ \\
\hline \multicolumn{12}{|l|}{ PR } \\
\hline Negative & 129 & 104 & 22 & \multicolumn{2}{|c|}{1.00 (reference) } & & & & & & \\
\hline Positive & 158 & 126 & 21 & $0.95^{\mathrm{m}}$ & $\begin{array}{l}0.99(0.70-1.40)^{\mathrm{m}} \\
0.78(0.41-1.48)^{\mathrm{n}}\end{array}$ & 0.78 & $\begin{array}{c}0.95 \\
(0.68-1.33)\end{array}$ & 0.44 & $\begin{array}{c}0.78 \\
(0.42-1.46)\end{array}$ & 0.58 & $\begin{array}{c}0.93 \\
(0.72-1.21)\end{array}$ \\
\hline
\end{tabular}

\section{HER-2}

\begin{tabular}{|c|c|c|c|c|c|c|c|c|c|c|c|}
\hline \multirow{3}{*}{$\begin{array}{l}\text { Negative } \\
\text { Positive }\end{array}$} & \multirow{3}{*}{$\begin{array}{l}192 \\
95\end{array}$} & \multirow{3}{*}{$\begin{array}{l}166 \\
64\end{array}$} & \multirow{3}{*}{$\begin{array}{l}31 \\
12\end{array}$} & \multicolumn{2}{|c|}{1.00 (reference) } & & \multirow{3}{*}{$\begin{array}{c}0.78 \\
(0.54-1.12)\end{array}$} & \multirow[b]{3}{*}{0.70} & \multirow{3}{*}{$\begin{array}{c}0.87 \\
(0.44-1.74)\end{array}$} & \multirow[b]{3}{*}{0.22} & \multirow{3}{*}{$\begin{array}{c}0.84 \\
(0.63-1.11)\end{array}$} \\
\hline & & & & $0.20^{\mathrm{m}}$ & $0.78(0.41-1.48)^{\mathrm{m}}$ & & & & & & \\
\hline & & & & $0.50^{\mathrm{n}}$ & $0.78(0.39-1.59)^{\mathrm{n}}$ & 0.18 & & & & & \\
\hline \multicolumn{12}{|c|}{ rs2978974 } \\
\hline \multicolumn{12}{|c|}{ Tumor size } \\
\hline$<2 \mathrm{~cm}$ & 89 & 87 & 12 & \multicolumn{2}{|c|}{1.00 (reference) } & & & & & & \\
\hline & & & & $0.73^{\mathrm{m}}$ & $1.07(0.74-1.54)^{\mathrm{m}}$ & & 1.12 & & 1.48 & & 1.14 \\
\hline$\geq 2 \mathrm{~cm}$ & 165 & 172 & 34 & $0.24^{\mathrm{n}}$ & $1.53(0.75-3.10)^{\mathrm{n}}$ & 0.52 & $(0.79-1.60)$ & 0.26 & $(0.75-2.93)$ & 0.34 & $(0.87-1.50)$ \\
\hline
\end{tabular}

\section{LN metastasis}

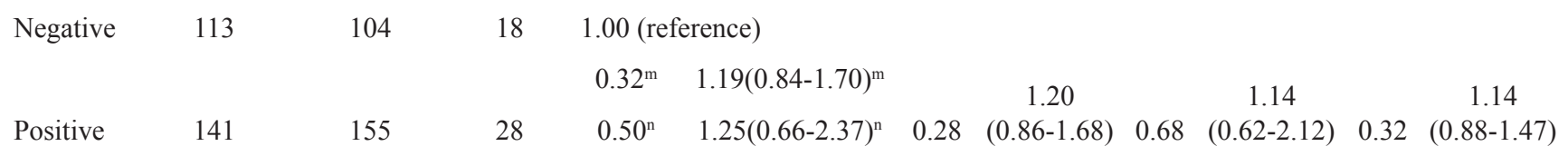




\begin{tabular}{|c|c|c|c|c|c|c|c|c|c|c|c|}
\hline \multirow[t]{2}{*}{ Variables } & \multirow[t]{2}{*}{$\mathbf{A A}$} & \multirow[t]{2}{*}{ Aa } & \multirow[t]{2}{*}{ aa } & \multicolumn{2}{|c|}{ Co-dominant } & \multicolumn{2}{|c|}{ Dominant } & \multicolumn{2}{|c|}{ Recessive } & \multicolumn{2}{|r|}{ Allele } \\
\hline & & & & $P$ & OR (95\%CI) & $P$ & $\begin{array}{c}\text { OR } \\
(95 \% \mathrm{CI})\end{array}$ & $P$ & $\begin{array}{c}\text { OR } \\
(95 \% C I)\end{array}$ & $P$ & $\begin{array}{c}\text { OR } \\
(95 \% \mathrm{CI})\end{array}$ \\
\hline \multicolumn{12}{|l|}{ ER } \\
\hline Negative & 108 & 121 & 18 & \multicolumn{2}{|c|}{1.00 (reference) } & & & & & & \\
\hline Positive & 146 & 138 & 28 & $0.34^{\mathrm{m}}$ & $0.84(0.60-1.20)^{\mathrm{m}}$ & 0.47 & $\begin{array}{c}0.88 \\
(0.63-1.24)\end{array}$ & 0.47 & $\begin{array}{c}1.25 \\
(0.68-2.33)\end{array}$ & 0.81 & $\begin{array}{c}0.97 \\
(0.75-1.25)\end{array}$ \\
\hline \multicolumn{12}{|l|}{ PR } \\
\hline Negative & 117 & 122 & 16 & \multicolumn{2}{|c|}{1.00 (reference) } & & & & & & \\
\hline Positive & 137 & 137 & 30 & $0.81^{\mathrm{m}}$ & $0.96(0.68-1.36)^{\mathrm{m}}$ & 0.85 & $\begin{array}{c}1.03 \\
(0.74-1.44)\end{array}$ & 0.12 & $\begin{array}{c}1.64 \\
(0.87-3.07)\end{array}$ & 0.43 & $\begin{array}{c}1.11 \\
(0.86-1.43)\end{array}$ \\
\hline \multicolumn{12}{|l|}{ HER-2 } \\
\hline Negative & 176 & 185 & 27 & \multicolumn{2}{|c|}{1.00 (reference) } & & & & & & \\
\hline Positive & 78 & 74 & 19 & $0.60^{\mathrm{m}}$ & $0.90(0.62-1.32)^{\mathrm{m}}$ & 0.96 & $\begin{array}{c}0.99 \\
(0.69-1.42)\end{array}$ & 0.10 & $\begin{array}{c}1.67 \\
(0.90-3.10)\end{array}$ & 0.52 & $\begin{array}{c}1.09 \\
(0.83-1.44)\end{array}$ \\
\hline
\end{tabular}

A: Major allele; a: Minor allele; $\mathrm{m}=$ Heterozygote model; $\mathrm{n}=$ Homozygote model; OR: odds ratio; 95\%CI: confidence interval; LN: lymph node; ER: estrogen receptor; PR: progesterone receptor; Her-2: human epidermal growth factor receptor-2.

Table 5: The haplotype frequencies of PSCA polymorphisms and breast cancer risk

\begin{tabular}{|c|c|c|c|c|c|c|}
\hline \multicolumn{3}{|l|}{ Haplotypes } & \multirow{2}{*}{$\begin{array}{c}\text { Controls }(\mathrm{N}=1166) \\
\mathrm{n}, \%\end{array}$} & \multirow{2}{*}{$\begin{array}{c}\text { Cases }(\mathrm{N}=1120) \\
\mathrm{n}, \%\end{array}$} & \multirow{2}{*}{$\begin{array}{c}\text { OR } \\
(95 \% \mathrm{CI})\end{array}$} & \multirow[t]{2}{*}{$p$} \\
\hline rs2294008 & rs2976392 & rs2978974 & & & & \\
\hline $\mathrm{C}$ & G & G & $526(45.12 \%)$ & $454(40.52 \%)$ & 1.00 (reference) & \\
\hline $\mathrm{C}$ & G & A & $317(27.17 \%)$ & $315(28.12 \%)$ & $1.15(0.94-1.41)$ & 0.168 \\
\hline $\mathrm{T}$ & A & G & $225(19.28 \%)$ & $296(26.39 \%)$ & $1.52(1.23-1.89)$ & $<0.001$ \\
\hline $\mathrm{T}$ & A & A & $22(1.90 \%)$ & $25(2.26 \%)$ & $1.32(0.73-2.37)$ & 0.357 \\
\hline Others & & & $76(6.52 \%)$ & $30(2.72 \%)$ & $0.46(0.29-0.71)$ & $<0.001$ \\
\hline
\end{tabular}

associated with an increased risk of lymph node metastasis. This study provides the first investigation of associations between rs 2978974 and breast cancer risk. We demonstrated that the minor allele of rs2978974 specifically increased the risk of breast cancer among postmenopausal women, while it was not associated with the risk of breast cancer among all patients, and was not associated with patient age or any of the clinicopathological features.

It is believed that haplotypes may be more important than any single SNP analysis in influencing a clinical response [33, 34]. To our knowledge, this is the first report of haplotypes in PSCA rs2294008, rs2976392, and rs2978974 polymorphisms. Haplotype analysis indicated that the $\mathrm{T}_{\mathrm{rs} 2294008} \mathrm{~A}_{\mathrm{rs} 2976392} \mathrm{G}_{\mathrm{rs} 2978974}$ haplotype was associated with significantly increased risk of breast cancer.

This study has several limitations. First, the singlecenter design may preclude extrapolation of our findings to other patient populations or ethnic groups. Second, we used a hospital-based case-control design, which may involve selection bias. Third, our sample size was relatively small, which may limit the strength of our stratified analyses. Fourth, we did not consider other important risk factors (e.g., high-dose radiation exposure at the chest, alcohol consumption, and other benign breast lesions), as we did not have access to these data. Therefore, a large well-designed prospective study is needed to validate our findings. Furthermore, biological function studies are crucial for elucidating the role of PSCA in breast cancer.

Our study revealed that the PSCA rs2294008 polymorphism influenced the risk of breast cancer among Chinese women and the rs2978974 polymorphism may specifically increase the risk of breast cancer in postmenopausal women. We found that rs2294008 was associated with PR-positive status and rs2976392 was associated with lymph node metastasis among Chinese women with breast cancer. Furthermore, the $\mathrm{T}_{\mathrm{rs} 2294008}$ $\mathrm{A}_{\mathrm{rs} 2976392} \mathrm{G}_{\mathrm{rs} 2978974}$ haplotype may increase the susceptibility to breast cancer. 


\section{MATERIALS AND METHODS}

\section{Study population}

We included the cases with pathologicallyconfirmed breast cancer, without history of any cancer, were treated at the Department of Oncology (Second Affiliated Hospital of Xi'an Jiaotong University) between January 2013 and October 2014. The healthy individuals who had visited the medical examination center at the Second Affiliated Hospital of Xi'an Jiaotong University for a check-up during the study period were included as controls. All individuals were Chinese Han women, and the controls were frequencymatched to the cases according to age ( \pm 5 years) and menopausal status. Finally, 560 eligible patients with an average age $49.09 \pm 11.02$ years and 583 healthy age-matched controls were included in the study (Table 6). The cases and controls exhibited similar clinical characteristics with the exception of body mass index (BMI) $(P=0.038)$.

A standardized epidemiological questionnaire was used to collect demographic and personal information.
Clinical information was collected from medical records and pathological reports. All participants were informed regarding the study's purpose and experimental procedures, and provided their written informed consent. The Human Research Committee at our institution approved the use of blood samples.

\section{SNP selection and genotyping}

Peripheral blood samples were collected in a standard tube and stored at $-80^{\circ} \mathrm{C}$. Genomic DNA was extracted from the peripheral whole blood samples using the Universal Genomic DNA Extraction Kit (version 3.0; TaKaRa, Japan). To achieve a power of at least $50 \%$, only SNPs with a minor allele frequency of $>0.01$ were included. Three primers were designed to amplify fragments of rs2294008, rs2978974, and rs2976392. Primers and PCR product sequences are shown in Table 7. DNA concentrations were measured by spectrometry (DU530 UV/VIS spectrophotometer; Beckman Instruments, Fullerton, CA, USA), Sequenom MassARRAY RS1000 was used for genotyping, and the related data were managed using Sequenom Typer 4.0 Software [35].

Table 6: The characteristics of breast cancer cases and cancer-free controls

\begin{tabular}{|c|c|c|c|c|}
\hline Characteristics & & Cases & Controls & $P$ \\
\hline Number & & 560 & 583 & \\
\hline Age $($ mean $\pm \mathrm{SD})$ & & $49.09 \pm 11.02$ & $48.80 \pm 8.28$ & 0.612 \\
\hline \multicolumn{5}{|l|}{ Menopausal status } \\
\hline Premenopausal & & 264 & 281 & \\
\hline Postmenopausal & & 296 & 302 & 0.716 \\
\hline \multicolumn{5}{|l|}{ Procreative times } \\
\hline$<2$ & & 289 & 291 & 0.594 \\
\hline$\geq 2$ & & 271 & 292 & \\
\hline \multicolumn{5}{|c|}{ Body mass index $\left(\mathrm{kg} / \mathrm{m}^{2}\right)$} \\
\hline$($ mean $\pm \mathrm{SD})$ & & $22.52 \pm 2.84$ & $22.95 \pm 3.21$ & 0.038 \\
\hline \multirow[t]{2}{*}{ Tumor size } & $<2 \mathrm{~cm}$ & 188 & & \\
\hline & $\geq 2 \mathrm{~cm}$ & 372 & & \\
\hline \multirow[t]{2}{*}{ LN metastasis } & Negative & 236 & & \\
\hline & Positive & 324 & & \\
\hline \multirow[t]{2}{*}{ ER } & Negative & 247 & & \\
\hline & Positive & 313 & & \\
\hline \multirow[t]{2}{*}{ PR } & Negative & 255 & & \\
\hline & Positive & 305 & & \\
\hline \multirow[t]{2}{*}{ Her-2 } & Negative & 389 & & \\
\hline & Positive & 171 & & \\
\hline
\end{tabular}

LN: lymph node; ER: estrogen receptor; PR: progesterone receptor; Her-2: human epidermal growth factor receptor-2. 
Table 7: Primers used for this study

\begin{tabular}{llll}
\hline SNP_ID & \multicolumn{1}{c}{ 1st-PCRP } & \multicolumn{1}{c}{ 2nd-PCRP } & \multicolumn{1}{c}{ UEP_SEQ } \\
\hline \multirow{2}{*}{ rs2294008 } & ACGTTGGATGTATAAAGT & ACGTTGGATGATCAACAGG & ccatGGCAAGCAGCACAGCCTTC \\
& CACCTGAGGCCC & GCAAGCAGCAC & \\
rs2976392 & ACGTTGGATGATCTTTCT & ACGTTGGATGAGATGCTG & GGAAGGAAAACAGCACA \\
& GGCCATCTGTCC & GGTGATTGTTGG & \\
rs2978974 & ACGTTGGATGTTGGACCCCA & ACGTTGGATGTCCCGGT & ggtGCAGTGCTGCCTTCC \\
& GCTAAGTAAG & GCAGTTTCTGATG & . \\
\hline
\end{tabular}

\section{Statistical analysis}

Microsoft Excel and SPSS software (version 21.0; SPSS Inc., Chicago, IL, USA) were used for all analyses. $P$-values were calculated using the $\chi^{2}$ test, and all tests were two-tailed; a $P$-value of $<0.05$ was considered statistically significant. The exact test was used to examine the distribution of each SNP among the controls, and their accordance with the HardyWeinberg equilibrium. Five different genetic models were used to evaluate the risk of breast cancer, with "A" used to indicate the major allele and "a" used to indicate the minor allele: the allele model (a vs. A); the co-dominant model (homozygote model: aa vs. AA; heterozygote model: Aa vs. AA); the recessive model (aa vs. $\mathrm{AA}+\mathrm{Aa}$ ); the dominant model (AA vs. $\mathrm{Aa}+\mathrm{aa}$ ); and the over-dominant model (AA+aa vs. Aa). The allelic frequencies for each SNP were compared between cases and controls in each model using the $\chi^{2}$ test and SNPStats software [36, 37]. Power calculations were made by PS software (Power and Sample Size Calculation, which was downloaded online: http://biostat.mc.vanderbilt. edu/wiki/Main/PowerSampleSize). Phase2.1 software was used to conduct all common haplotypes [34] and SPSS software was used to estimate the ORs and $95 \%$ CIs for each haplotype. As shown in Table 6, there was a significant difference in BMI between breast cancer cases and controls $(P=0.038)$. BMI may be a confounder in the development of breast cancer. Therefore, to control for its effects, we used stratification analyses. First, we calculated OR1 and OR2 by stratification for BMI. Then, the ratio of the OR (unadjusted OR) and OR1/OR2 was calculated. If the ratio was close to 1 , the results did not need to be adjusted, indicating that BMI was not a confounder. Otherwise, we would need to adjust results for BMI.

\section{ACKNOWLEDGMENTS}

This study was supported by National Natural Science Foundation, China (No. 81471670; 81274136); China Postdoctoral Science Foundation (No. 2014M560791; 2015T81037); the Fundamental Research Funds for the Central Universities, China (No. 2014qngz-04); the
International Cooperative Project (No. 2013KW-32-01) and Science and Technology Plan of Innovation Project, Shaanxi province, China (No. 2015KTCL03-06). We also thanks for the language editing by Editage.

\section{COMPETING INTERESTS}

The authors have declared that no competing interest exists.

\section{REFERENCES}

1. Zeng H, Zheng R, Zhang S, Zou X, Chen W. Female breast cancer statistics of 2010 in China: estimates based on data from 145 population-based cancer registries. J Thorac Dis. 2014; 6:466-470.

2. Chen W, Zheng R, Zeng H, Zhang S, He J. Annual report on status of cancer in China, 2011. Chin J Cancer Res. 2015; 27:2-12.

3. Lichtenstein P, Holm NV, Verkasalo PK, Iliadou A, Kaprio J, Koskenvuo M, Pukkala E, Skytthe A, Hemminki $\mathrm{K}$. Environmental and heritable factors in the causation of cancer-analyses of cohorts of twins from Sweden, Denmark, and Finland. N Engl J Med. 2000; 343:78-85.

4. Ellsworth RE, Decewicz DJ, Shriver CD, Ellsworth DL. Breast Cancer in the Personal Genomics Era. Curr Genomics. 2010; 11: 146-161.

5. Fackenthal JD, Olopade OI. Breast cancer risk associated with BRCA1 and BRCA2 in diverse populations. Nat Rev Cancer. 2007; 7: 937-948.

6. Fanale D, Amodeo V, Corsini LR, Rizzo S, Bazan V, Russo A. Breast cancer genome-wide association studies: there is strength in numbers. Oncogene. 2012; 31: 2121-2128.

7. Katoh M. Cancer genomics and genetics of FGFR2 (Review). Int J Oncol. 2008; 33: 233-237.

8. Yu KD, Di GH, Fan L, Chen AX, Yang C, Shao ZM. Lack of an association between a functional polymorphism in the interleukin- 6 gene promoter and breast cancer risk: a meta-analysis involving 25,703 subjects. Breast Cancer Res Treat. 2010; 122:483-488.

9. Kim SY, Yoo JY, Shin A, Kim Y, Lee ES, Lee YS. Prostate stem cell antigen single nucleotide polymorphisms 
influence risk of estrogen receptor negative breast cancer in Korean females. Asian Pac J Cancer Prev. 2012; 13: 41-48.

10. Reiter RE, Gu Z, Watabe T, Thomas G, Szigeti K, Davis E, Wahl M, Nisitani S, Yamashiro J, Le Beau MM, Loda M, Witte ON. Prostate stem cell antigen: a cell surface marker overexpressed in prostate cancer. Proc Natl Acad Sci USA. 1998; 95:1735-40.

11. Gu Z, Thomas G, Yamashiro J, Shintaku IP, Dorey F, Raitano A, Witte ON, Said JW, Loda M, Reiter RE. Prostate stem cell antigen (PSCA) expression increases with high gleason score, advanced stage and bone metastasis in prostate cancer. Oncogene. 2000; 19: 1288-1296.

12. Fu YP, Kohaar I, Rothman N, Earl J, Figueroa JD, Ye Y, Malats N, Tang W, Liu L, Garcia-Closas M, Muchmore B, Chatterjee N, Tarway M, et al. Common genetic variants in the PSCA gene influence gene expression and bladder cancer risk. Proc Natl Acad Sci USA. 2012; 109:4974-4979.

13. Wang $\mathrm{P}$, Ye D, Guo J, Liu F, Jiang H, Gong J, Gu C, Shao Q, Sun J, Zheng SL, Yu H, Lin X, Xia G, et al. Genetic score of multiple risk-associated single nucleotide polymorphisms is a marker for genetic susceptibility to bladder cancer. Genes Chromosomes Cancer. 2014; 53: 98-105.

14. Ma Z, Hu Q, Chen Z, Tao S, Macnamara L, Kim ST, Tian L, Xu K, Ding Q, Zheng SL, Sun J, Xia G, Xu J. Systematic evaluation of bladder cancer risk-associated single-nucleotide polymorphisms in a Chinese population. Mol Carcinog. 2013; 52:916-921.

15. Lochhead P, Frank B, Hold GL, Rabkin CS, Ng MT, Vaughan TL, Risch HA, Gammon MD, Lissowska J, Weck MN, Raum E, Müller H, Illig T, et al. An association between a variation in the PSCA gene and upper gastrointestinal cancer in Caucasians. Gastroenterology. 2011; 140: 435-441.

16. Gu X, Zhang W, Xu L, Cai D. Quantitative assessment of the influence of prostate stem cell antigen polymorphisms on gastric cancer risk. Tumour Biol. 2014; 35: 2167-2174.

17. Zhao J, Geng P, Li Z, Cui S, Zhao J, Wang L, Li J, Ji F, Li G, Shen G, Lin M, Shen C. Prostate stem cell antigen rs2294008 polymorphism differentially contributes to Helicobacter pylori-negative gastric cancer among various populations in China. Mol Clin Oncol.2013; 1: 493-498.

18. Elsamman EM, Fukumori T, Tanimoto S, Nakanishi R, Takahashi M, Toida K, Kanayama HO. The expression of prostate stem cell antigen in human clear cell renal cell carcinoma: a quantitative reverse transcriptase polymerase chain reaction analysis. BJU Int. 2006; 98:668-673.

19. Dai N, Zheng M, Wang C, Ji Y, Du J, Zhu C, He Y, Zhu M, Zhu X, Sun M, Dai J, Ma H, Chen J, et al. Genetic variants at $8 \mathrm{q} 24$ are associated with risk of esophageal squamous cell carcinoma in a Chinese population. Cancer Sci. 2014; 105: 731-735.

20. Wang P, Ye D, Guo J, Liu F, Jiang H, Gong J, Gu C, Shao Q, Sun J, Zheng SL, Yu H, Lin X, Xia G, et al. Genetic score of multiple risk-associated single nucleotide polymorphisms is a marker for genetic susceptibility to bladder cancer. Genes Chromosomes Cancer. 2014; 53: 98-105.

21. Ono H, Chihara D, Chiwaki F, Yanagihara K, Sasaki H, Sakamoto H, Tanaka H, Yoshida T, Saeki N, Matsuo K. Missense allele of a single nucleotide polymorphism rs2294008 attenuated antitumor effects of prostate stem cell antigen in gallbladder cancer cells. J Carcinog. 2013; 12:4.

22. Rai R, Sharma KL, Misra S, Kumar A, Mittal B. PSCA gene variants (rs2294008 and rs2978974) confer increased susceptibility of gallbladder carcinoma in females. Gene. 2013; 530:172-177.

23. Grubbs EG, Abdel-Wahab Z, Tyler DS, Pruitt SK. Utilizing quantitative polymerase chain reaction to evaluate prostate stem cell antigen as a tumor marker in pancreatic cancer. Ann. Surg. Oncol. 2006; 13:1645-1654.

24. Study Group of Millennium Genome Project for Cancer. Genetic variation in PSCA is associated with susceptibility to diffuse-type gastric cancer. Nat. Genet. 2008; 40(6):730-40.

25. Marra E, Uva P, Viti V, Simonelli V, Dogliotti E, De Rinaldis E, Lahm A, La Monica N, Nicosia A, Ciliberto G, Palombo F. Growth delay of human bladder cancer cells by Prostate Stem Cell Antigen downregulation is associated withactivation of immune signaling pathways. B.M.C Cancer. 2010; 10:129.

26. Wang M, Wang XJ, Ma YF, Ma XB, Dai ZM, Lv Y, Lin S, Liu XH, Yang PT, Dai ZJ. PSCA rs2294008 C > $\mathrm{T}$ polymorphism contributes to gastric and bladder cancer risk. Ther Clin Risk Manag. 2015; 11: 237-245.

27. Gu Y, Dai QS, Hua RX, Zhang B, Zhu JH, Huang JW, Xie BH, Xiong SQ, Tan GS, Li HP. PSCA s2294008 C>T and rs2976392 G>A polymorphisms contribute to cancer susceptibility: evidence from published studies. Genes Cancer. 2015; 6:254-264. doi: 10.18632/genesandcancer.63.

28. Saeki N, Gu J, Yoshida T, Wu X. Prostate stem cell antigen: a Jekyll and Hyde molecule. Clin Cancer Res. 2010; 16:3533-3538.

29. Wang M, Chu H, Lv Q, Wang L, Yuan L, Fu G, Tong N, Qin C, Yin C, Zhang Z, Xu J. Cumulative effect of genome-wide association study-identified genetic variants for bladder cancer. Int J Cancer. 2014; 135: 2653-2660.

30. Zhao J, Geng P, Li Z, Cui S, Zhao J, Wang L, Li J, Ji F, Li G, Shen G, Lin M, Shen C. Prostate stem cell antigen rs2294008 polymorphism differentially contributes to Helicobacter pylori-negative gastric cancer among various populations in China. Mol Clin Oncol. 2013; 1:493-498.

31. Rizzato C, Kato I, Plummer M, Muñoz N, Canzian F. Genetic variation in PSCA and risk of gastric advanced preneoplastic lesions and cancer in relation to Helicobacter pylori infection. PLoS One. 2013; 8:e73100.

32. Chandra V, Kim JJ, Gupta U, Mittal B, Rai R. Impact of DCC (rs714) and PSCA (rs2294008 and rs2976392) 
Gene Polymorphism in Modulating Cancer Risk in Asian Population. Genes (Basel). 2016; 7: pii: E9.

33. Chu CM, Chen CJ, Chan DC, Wu HS, Liu YC, Shen CY, Chang TM, Yu JC, Harn HJ, Yu CP, Yang MH. CDH1 polymorphisms and haplotypes in sporadic diffuse and intestinal gastric cancer: a case-control study based on direct sequencing analysis. World J Surg Oncol. 2014; 12: 80.

34. Cheng XL, Ning T, Xu CQ, Li Y, Zhu BS, Hou FT, Zhang SY, Chen ZP. Haplotype analysis of CTLA4 gene and risk of esophageal squamous cell carcinoma in Anyang area of China. Hepatogastroenterology. 2011; 58:432-437.
35. Thomas RK, Baker AC, Debiasi RM, Winckler W, Laframboise T, Lin WM, Wang M, Feng W, Zander T, MacConaill L, Lee JC, Nicoletti R, Hatton C, et al. Highthroughput oncogene mutation profiling in human cancer. Nat Genet. 2007; 39: 347-351.

36. Solé X1, Guinó E, Valls J, Iniesta R, Moreno V. SNPStats: a web tool for the analysis of association studies. Bioinformatics. 2006; 22: 1928-1929.

37. Adamec C. Example of the use of the nonparametric test: Test $\chi 2$ for comparison of 2 independent examples. Cesk Zdrav. 1964; 12: 613-619. 